\title{
Die COVID-Impfung und der Merkurstab
}

\section{Jana Siroka}

Dr. med., Mitglied des Zentralvorstands der FMH und Departementsverantwortliche Stationäre Versorgung und Tarife

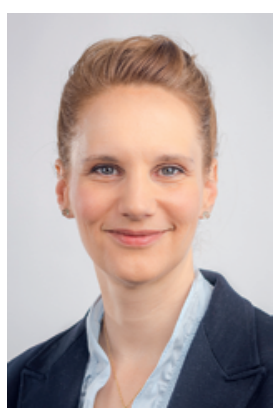

Seit Jahrtausenden sind der Stab des Äskulap mit einer Schlange und der Merkurstab mit zwei Schlangen Symbole unseres ärztlichen Berufsstandes. Der Merkurstab, um den sich eine schwarze und eine weisse Schlange winden, ist ein Symbol der Erkenntnis, des Umgangs mit Polaritäten. Er symbolisiert die Fähigkeit zur merkuriellen, geflügelten Beweglichkeit zwischen zwei Polen.

Sei es im Abwägen von Therapieoptionen, im sozialen Umgang mit Patienten und Angehörigen, im interprofessionellen Zusammenspiel ... immer wieder ist von uns ein vernünftiges Bewegen zwischen den Polen gefragt.

Heute stehen wir als Ärzteschaft mitten in einer medizinischen und gesellschaftlichen Krise - der COVID-Krise. Damit verbinden sich Fragen zu den medizinischen Therapien und Behandlungsoptionen, zum Beispiel auch Fragen über die COVID-Impfung. Aufgrund unseres medizinischen Wissens und unserer Erfahrung können wir antizipieren, dass die Impfungen alleine uns wahrscheinlich nicht aus dieser Krise herausführen werden. Vernünftigerweise sollten wir auch in der Pandemie die Pole nicht aus den Augen verlieren. Wir haben als den einen Pol die Prävention, also die Möglichkeit der potenziellen Verhinderung ei-

\section{Heute stehen wir als Ärztinnen und Ärzte mitten in einer medizinischen und gesell- schaftlichen Krise - der COVID-Pandemie.}

ner Erkrankung an COVID-19. Eine gute Immunität als Prävention vor Erkrankung kann ausser durch eine gesunde Lebensführung auch mit einer COVID-Impfung erlangt werden. An dieses Vorgehen sind wie bei allen anderen Impfungen auch Fragen der Sicherheit und Wirksamkeit geknüpft: Wie lange wird die Impfung wirken? Wie oft muss sie wiederholt werden? Wie oft kann sie wiederholt werden? Wie ist es mit seltenen oder späten Nebenwirkungen? Wie ist es bei VirusMutationen? Dann gibt es aber auch Menschen, die an COVID-19 erkrankt und davon genesen sind. Auch hier stellen sich bezüglich der daraus resultierenden Immunität ähnliche Fragen wie bei der Impfung: Wie lange hält der Schutz? Wie ist es bei Virus-Mutationen? Bei SARS-CoV-1 weiss man, dass die zellulär vermittelte Immunität über viele Jahre erhalten bleibt; bei SARSCoV-2 fehlen aus naheliegenden Gründen die Daten

Vernünftigerweise sollten wir als Mediziner auch jetzt unsere beiden Pole nicht aus den Augen verlieren: das Vorbeugen und das Heilen.

zur Dauer der natürlich erworbenen Immunität. Bisher liegen keine validen Daten vor, ob COVID-Impfungen kurz nach effektiv durchgemachter Erkrankung an COVID-19 schwerere Impf-Nebenwirkungen hervorrufen können. Entsprechend heterogen und volatil sind die Empfehlungen der einzelnen Länder, wie damit umzugehen ist.

Und dann gibt es den anderen Pol. Nicht die Prävention, sondern die Behandlung. Therapien suchen und finden, um diese Krankheit mit ihren zum Teil schweren oder chronischen Verläufen zu behandeln, bestenfalls zu heilen. Hier frage ich mich, ob wir diesen Pol, nämlich die Behandlung der Krankheit, in der medialen Impf-Euphorie nicht zu sehr aus den Augen verloren haben. Ich hoffe, wir Ärztinnen und Ärzte werden mit aller Kraft, mit Forschergeist und Heilerwillen versuchen, Therapien und Heilmittel für Menschen zu finden, die an COVID erkrankt sind.

Denn zur Überwindung der COVID-Krise braucht es eben beides, Prävention und Heilung - symbolisiert durch die beiden Schlangen des Merkurstabs. 